\title{
Lesão contusa de artéria ilíaca comum esquerda por cinto de segurança
}

\author{
Blunt injury to the left common iliac artery caused by seat belt
}

\section{Fábio M esquita de Souza', Wagner Rodrigues C hagas², Luis Roberto Velloso ${ }^{3}$, Jonas M arques Ribas ${ }^{4}$}

\begin{abstract}
Resumo
Os autores relatam um caso de paciente de 17 anos, vítima de acidente automobilístico com impacto frontal, que apresentava, ao socorro médico, dor abdominal intensa e tatuagem transversa, em região infra-umbilical, causada pelo cinto de segurança. Foi submetida a laparotomia exploradora, que evidenciou pequena lesão lacerante de jejuno terminal, não sendo encontradas outras lesões à inspeção. Ao término da cirurgia, verificou-se cianose moderada em região plantar esquerda, com ausência de pulsos tibiais e poplíteos. 0 pulso da artéria femoral encontrava-se diminuído. A arteriografia realizada mostrou obstrução do fluxo em artéria ilíaca comum esquerda. A re construção foi realizada com interposição de prótese de $\mathrm{D}$ acron de 6 $\mathrm{mm}$.
\end{abstract}

Palavras-chave: trauma, reconstrução endoluminal, cirurgia.

\begin{abstract}
The authors report a case of a 17-year-old female patient involved in a head-on motor vehicle collision. The patient presented to the emergency department complaining of severe abdominal pain. She also had a transverse bruise in the inferior anterior abdominal wall, suggesting a seat-belt sign. The patient underwent an exploratory laparotomy, which showed a small distal jejunal perforation. Other lesions were not found at inspection. At the end of the operation, moderate cyanosis of the plantar aspect of the left foot was observed, and popliteal and distal pulses were absent. The intensity of the femoral pulse was decreased. An arteriography revealed obstruction of the left common iliac artery. Repair was successfully achieved with a 6-mm Dacron graft interposition.
\end{abstract}

Key words: trauma, endoluminal repair, surgery.

D evido à infreqüência dessetipo de lesão, 0 atendimento inicial bem realizado é fundamental para 0 diagnóstico precoce. A arteriografia ou eco-D oppler devem ser realizados sempre que houver suspeita de lesão vascular ${ }^{3}$.

\section{Relato do caso}

Paciente feminina, 17 anos, vítima de acidente automobilístico com impacto frontal, deu entrada no pronto socorro, consciente, queixando-se de dor abdominal forte, dor no antebraço esquerdo e dor lombar.

Ao exame físico, verificou-se fratura de antebraço esquerdo, que foi imobilizado, bem como escoriação transversal em região infra-umbilical (cinto de segurança de três pontos) (Figura 1). 0 exame radiográfico revel ou fratura da primeira vértebra lombar e a necessidade delaparotomia exploradora, quefoi realizada pela equipe de cirurgia geral de plantão. A exploração da cavidade abdominal mostrou pequena lesão em jejuno 
terminal, provavelmente causada por laceração pela compressão da alça jejunal entre o cinto de segurança e a coluna lombar (mecanismo compressão/desaceleração). Essa lesão foi tratada com sutura primária. 0 músculo grande reto abdominal do lado esquerdo encontrava-se com laceração extensa desde a cicatriz umbilical até sua borda lateral, mostrando claramente a participação do cinto de segurança no mecanismo do trauma de parede abdominal, da artéria ilíaca comum e da alça jejunal. N ão se observaram, à inspeção da cavidade, outras lesões dignas de nota, nem mesmo hematomas no mesocólon ou mesosigmóide. Ao fim do procedimento cirúrgico, quando a paciente foi reavaliada, percebeu-se moderada cianose em região plantar esquerda, diminuição da temperatura em todo o membro, ausência de pulsos tibiais e poplíteos e diminuição importantedo pulso femoral. 0 membro inferior direito apresentava-se sem alterações.

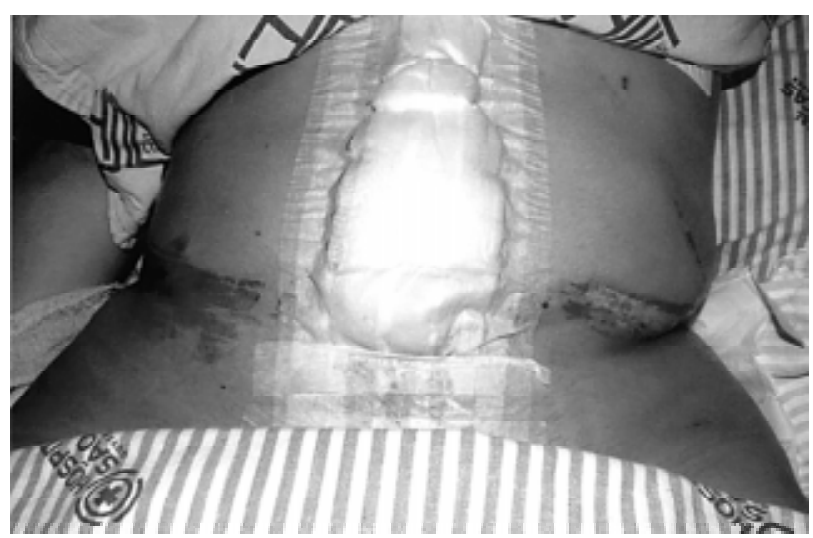

Figura 1 - T atuagem causada pelo cinto de segurança

Foi realizada arteriografia, indicada pela equipe de cirurgia vascular, por punção de artéria femoral contralateral, que revelou oclusão de artéria ilíaca comum esquerda (Figura 2).

A paciente foi reencaminhada ao bloco cirúrgico e submetida a reabertura da cavidade abdominal para correção da lesão, uma vez que não temos, em nossa cidade, serviço decirurgia en dovascular, o que pouparia esse novo procedimento. A exploração do retroperitônio, à esquerda, mostrou segmento de artéria ilíaca

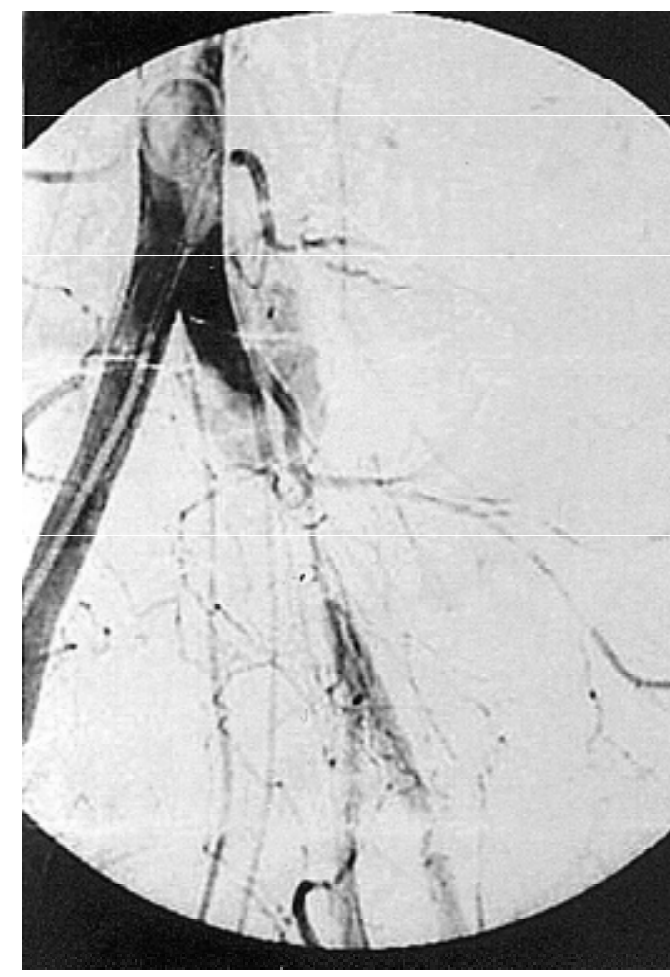

Figura 2 - Lesão de artéria ilíaca comum esquerda

comum trombosado e sem pulso distal. A aorta infrarenal apresentava-se sem alterações.

Foi realizada ressecção desse segmento e interposto prótese de Dacron $6 \mathrm{~mm}$, com anastomose términoterminal ilíaco-ilíaco proximal edistal. Antes do término da anastomose distal, realizamos a passagem do cateter de Fogarty, não encontrando coágulos.

A abertura do segmento arterial lesado revelou ruptura e dissecção da camada íntima posterior, em sentido transversal, com flap intimal projetando-separa a luz arterial, provocando a oclusão.

Ao se liberar os clampes, houve bom pulso em artéria ilíaca externa e interna. N ão houve, porém, pulsos poplíteos etibiais. 0 pulso femoral encontravaseainda diminuído. U ma arteriografia intra-operatória mostrou afilamento de toda árvore arterial no membro inferior, interpretado como intenso vasoespasmo resultante da agressão pel o cateter deFogarty (Figura 3A). A injeção de solução de papaverina, utilizando o próprio cateter deF ogarty com o balão roto, nospareceu recurso viável, pois poderíamosal cançar toda a extensão arterial desejada e já o tínhamos na mesa cirúrgica. De fato, 
mostrou-se extremamente eficaz, como pode ser comprovado na arteriografia de controle, revertendo o vasoespasmo (Figura 3B). O s pulsos tibiais e poplíteos eram então perfeitamente palpáveis. A síntese da cavidade foi realizada de modo padrão. bem.

A paciente encontra-se em recuperação e evolui

\section{D iscussão}

O sferimentos contusos são produzidos por traumatismos fechados e são responsáveis por $10 \%$ das lesões vasculares, as quais ocorrem geralmente nos

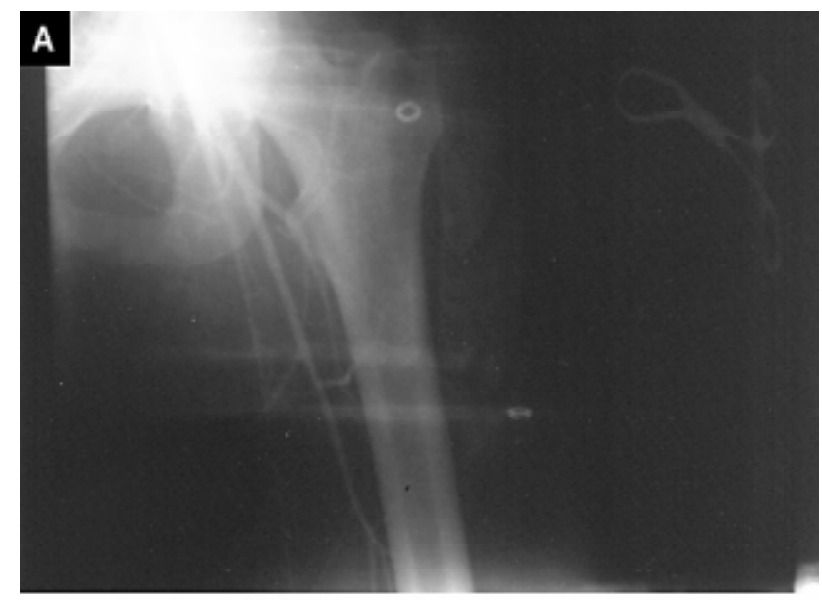

B

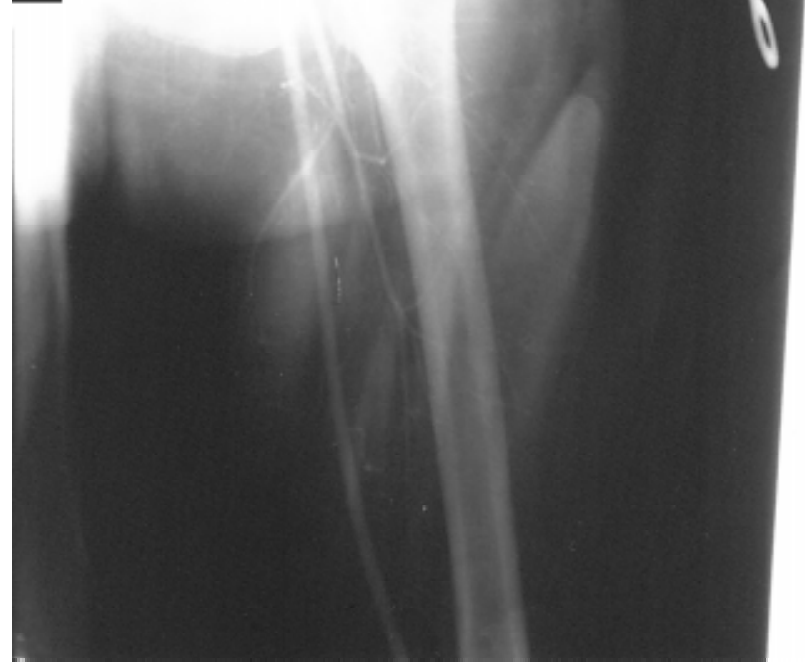

Figura 3 - (A) Intenso vasoespasmo da artéria femoral superficial, provavelmentecausado pelo cateter deFogarty. (B) Artériafemoral superficial após injeção de solução de papaverina locais onde os vasos estão em íntimo contato com estruturas osteoarticulares ${ }^{4}$. A condição para a lesão arterial tem sido associada a dois tipos diferentes de força criada durante o impacto (mecanismo de compressão/desaceleração), o que produz um forte esmagamento, causando descontinuidade da parede vascular e/ou formação de flap intimal. Alguns autores observaram que a lesão vascular tem ocorrido mais freqüentemente do mesmo lado (esquerdo) da fixação do cinto de segurança, onde a força de esmagamento é mais intensa devido à junção entre a alça transversal e a diagonal ${ }^{1}$. Esse mecanismo também poderia explicar a lesão intestinal.

0 trauma vascular podeser facilmente diagnosticado na presença dehemorragia externa volumosa, hematoma pulsátil, pequenos hematomas no mesocólon e mesosigmóide, ausência de pulso distal ou sinais evidentes de isquemia. No caso relatado, a ausência de pulsos poplíteos e tibiais e a diminuição do pulso femoral à esquerda não foram inicial mente observadas, o que retardou o tratamento eobrigou a uma reabertura de cavidade para correção da lesão. 0 retroperitônio não foi explorado, uma vez que não havia indícios para tal. É fundamental quetoda vítima de traumatismo seja considerada um politraumatizado, eque seja obedecida uma rígida epadronizada seqüência deavaliação inicial .

$\mathrm{N}$ a literatura, encontramos um caso de lesão de artéria ilíaca comum, causada por cinto de segurança, diagnosticada após 3 meses da lesão vascular, quando foi realizada arteriografia para investigação de claudicação e diminuição de pulso femoral ${ }^{1}$. I sso mostra que não é incomum essas lesões passarem despercebidas.

Em levantamento de traumas vasculares em 97 pacientes com 106 lesões arteriais, sendo 53 civis e 44 traumatizados de guerra, não houve relato de lesões de artéria ilíaca comum ${ }^{5}$.

Em outro levantamento com 25 pacientes com 32 traumas vasculares abdominopélvicos, apenas três foram de artéria ilíaca comum ${ }^{6}$. N a literatura pesquisada, encontramos, como descrito acima, poucos casos de lesão contusa de artéria ilíaca comum, citadas como raro fenômeno, eapenas um caso de lesão contusa desta mesma artéria causada por cinto de segurança².

N o caso relatado, optamos por imediata intervenção, uma vez quea paciente apresentava sinais claros de déficit circulatório no membro inferior esquerdo. A transposição da artéria comum esquerda, anastomosando a artéria comum direita, o reimplante da ilíaca 
interna contralateral ou o reimplante da ilíaca interna ipsilateral são técnicas possíveis de serem realizadas. Porém, nenhuma nos pareceu uma boa opção, pois além deestender o tempo cirúrgico e, portanto, o tempo de isquemia, o risco de lesão venosa é al to. Consideramos o uso da prótese uma opção adequada, visto que o calibre da artéria lesada era incompatível com a veia safena, e os resultados em longo prazo são semel hantes para esse segmento.

0 tratamento endovascular para o trauma contuso éinfreqüente. Entretanto, em caso relatado de paciente de 27 anos, politraumatizado, com lesão contusa bilateral de artérias ilíacas, houve sucesso com tratamento endovascular bilateral. Em nosso caso, não foi usado esse recurso, pois não o temos disponível. N este trabaIho, conclui-seque o reparo endovascular para traumas vasculares contusos é possível e deve ser considerado quando há contaminação fecal, hematoma pélvico, ou múltiplas lesões que tornam o reparo convencional problemático ${ }^{7}$. 0 tratamento endovascular é 0 mais recente e significativo avanço no manejo de lesões vasculares muito complexas 4 .

O s avanços no tratamento do paciente politraumatizado e o diagnóstico e tratamento imediatos das lesões vasculares têm permitido uma melhora progressiva nos resultados ${ }^{4}$.

\section{R eferências}

1. D ell'Erba A, Di Vella G, G iardino N. Seatbelt injury to the common iliac artery: casereport.J ForensicSci. 1998;43:215-7.

2. Gupta N, Auer A, Troop B. Seat belt-related injury to the common iliac artery: case report and review of the literature. J Trauma. 1998;45:419-21.

3. Tuec JJ, Villapadierna F, Singland JD, Papon X, Pessaux P, $V$ ergos M . Blunt injury to the common iliac artery. Eur J V asc Endovasc Surg. 2000;20:47-50.

4. M affei $F H$. Traumatismos vasculares. In: $M$ affei $F H, L$ astória $S$, Yoshida W B, Rollo H A. D oenças vasculares periféricas. 3 a ed. São Paulo: M edsi; 2002. p. 1669-92.

5. Velinovic M M , D avidovic BL, LotinalS, et al. Complications of operative treatment of injuries of peripheral arteries. Cardiovasc Surg. 2000;8:256-64.

6. Sriussadaporn $\mathrm{S}$. Abdominopelvic vascular injuries. J M ed Assoc Thai. 2000;83:13-20.

7. Sternbergh WC, Conners M S, O jeda M A, M oney SR. Acute bilateral iliac artery occlusion secondary to blunt trauma: successful endovascular treatment. J Vasc Surg. 2003;38: 589-92.

Correspondência:

Fábio M esquita de Souza

Rua Barão do Rio Branco, 681/304, Centro

CEP 35010-030 - Governador V aladares, M G

Tel.: (33) 3271.6510/3272.7979

E-mail: fabiomeskita@ig.com.br 